\title{
CONFIANZA EN EL GOBIERNO LOCAL Y CAPACIDADES INSTITUCIONALES DE LOS MUNICIPIOS CHILENOS: UN ANÁLISIS MULTINIVEL
} 4

\author{
TRUST IN LOCAL GOVERNMENT
}

AND INSTITUTIONAL CAPACITIES

OF THE CHILEAN MUNICIPALITIES:

A MULTILEVEL ANALYSIS

\section{Claudio Fuentes González}

Recibido: 07/11/2018

Aceptado: 14/12/2018

\section{RE S U M E N}

Las municipalidades son consideradas como la primera cara del Estado para los ciudadanos. A su vez, los gobiernos locales presentan mayores nivel de confianza en comparación con otras instituciones políticas. A pesar de ello, los estudios sobre las municipalidades no son tan abundantes como otros en la ciencia política chilena. En este sentido, existen casi nulos estudios que vinculen la confianza política y las capacidades institucionales a nivel local. Sumado a ello, dicha escasez es aún mayor cuando se consideran diversos niveles de medición para el examen de fenómenos políticos a escala local y de las personas. Para contribuir con cubrir lentamente esas brechas, la pregunta de investigación de este estudio es si las capacidades institucionales de las municipalidades inciden en la confianza política que las personas depositan en ellas y, de existir tal influencia, cómo es dicha relación. Para dar respuesta a dicha interrogante, se desarrolla un estudio cuantitativo con el objetivo de testear desde un nivel contextual, el municipio, el efecto de las capacidades institucionales (administrativa-técnica; extractiva/fiscal, y política) sobre las percepciones ciudadanas referentes a la confianza en la municipalidad. Dado que se combinan dos niveles de medición, el individual y el comunal, se propone un análisis multinivel con 
datos recogidos desde el Proyecto de Opinión Pública de América Latina (LAPOP) 2016, datos electorales oficiales y datos del sistema de información municipal chileno. Las principales conclusiones son que las capacidades institucionales tienen efectos sobre la confianza en los gobiernos locales desde una dinámica multinivel, y que la interacción entre las distintas dimensiones de la capacidad institucional tiene efectos moderadores. Es decir, las capacidades institucionales de las municipalidades, en tanto contexto, afectan las percepciones, actitudes y comportamientos de los ciudadanos.

\section{PA L A R A S C L V E}

Confianza, Municipalidad, Gobierno Local, Capacidad Institucional, Multinivel, Apoyo Político.

\section{A B S T R A C T}

Municipalities are considered as the first face of the State for the citizens. At the same time, local governments have a higher level of confidence compared to other political institutions. Despite this, studies on municipalities are not as abundant as others in Chilean political science. In this sense, there are almost no studies that link political trust and institutional capacities at the local level. Moreover, this scarcity is even greater when different levels of measurement are taken into account for the examination of political phenomena at the local scale and for people. To contribute to slowly filling these gaps, the research question in this study is whether the institutional capacities of municipalities influence the political trust that people place in them and, if such influence exists, what that relationship is like. In order to answer this question, a quantitative study is developed with the objective of testing from a contextual level, the municipality, the effect of institutional capacities (administrative-technical; extractive/fiscal, and political) on citizen perceptions referring to trust in the municipality. Given that two levels of measurement are combined, the individual and the communal, a multilevel analysis is proposed with data collected from the Latin American Public Opinion Project (LAPOP) 2016, official electoral data and data from the Chilean municipal information system. The main conclusions are that institutional capacities have effects on trust in local governments from a multi-level dynamic, and that the interaction between the different dimensions of institutional capacity has moderating effects. In other words, the institutional capacities of municipalities, as a context, affect the perceptions, attitudes and behaviours of citizens.

\section{K E Y W O R D}

Trust, Municipality, Local Government, Institutional Capacity, Multilevel, Political Support. 


\section{I N T R O D U C C I Ó N}

Para Dalton, las "encuestas de opinión pública generalmente indican que los ciudadanos confían más en los gobiernos locales que en su gobierno nacional" (2004:203). Esto es refrendado por el Centro de Políticas Públicas UC (2016a), quienes sostienen que las municipalidades son más próximas a las personas, son la primera cara del Estado en el territorio y es la institución pública que presenta los mayores niveles de confianza. Para el caso chileno, ambas afirmaciones son correctas. El gráfico 1 evidencia que, en términos comparados, la confianza en la municipalidad está por sobre otras instituciones políticas.

El gráfico 1 nos permite hacer algunas reflexiones. Primero, las municipalidades son las que muestran mayores niveles de confianza en comparación a otras instituciones políticas. Segundo, su tendencia es plana y todas las demás son a la baja. Esto se explicaría por la estabilidad de la medición y baja dispersión. Tercero, pareciera ser que las masivas movilizaciones sociales de 2010-2012 no afectaron a la confianza en las municipalidades. Cuarto, los $\mathrm{r}$ cuadrados de las líneas de tendencia de cada curva se empinan casi todas por encima del 0,47 ; salvo la confianza en la municipalidad que logra un $r$ cuadrado de 0,001. En simple, esto significaría que el paso del tiempo no afecta a la confianza en el gobierno local. En consecuencia, pareciera ser que la confianza en la municipalidad se ve afectada por otras variables en comparación a las otras instituciones, o que sus dinámicas institucionales la diferencian de las demás instituciones. Esas "otras" variables en este estudio se entienden como las capacidades institucionales de los municipios, factores que no han sido puestos aprueba, al menos en Chile, aún. 


\section{GRÁFICO 1: COMPARACIÓN DE NIVEL DE CONFIANZA DE INSTITUCIONES POLÍTICAS EN CHILE, 2006-2016}

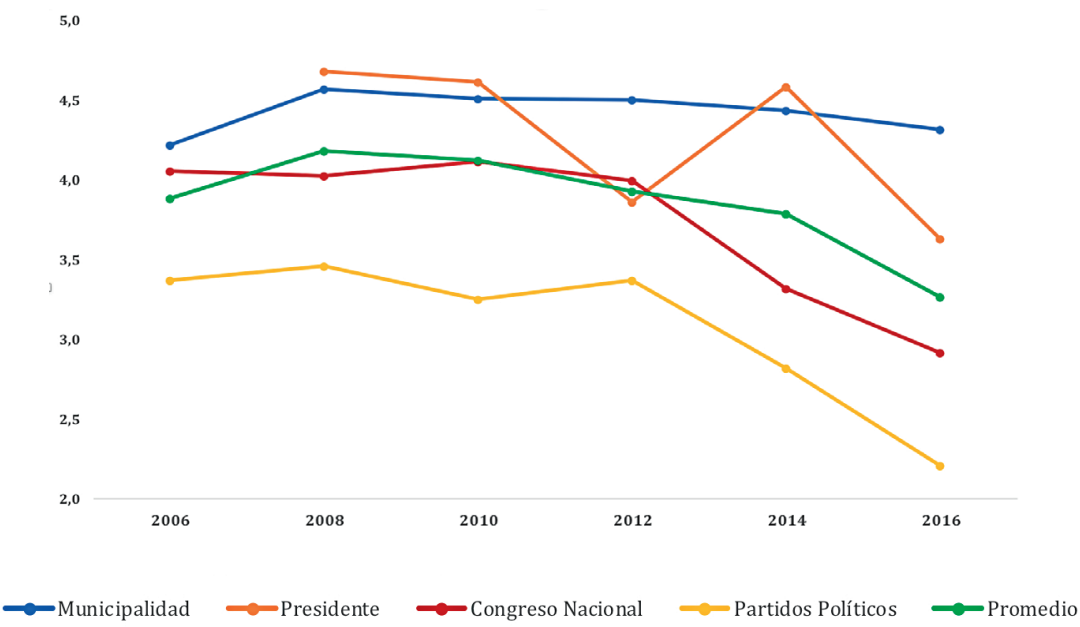

FUENTE: ELABORACIÓN PROPIA CON DATOS DE LAPOP 2006, 2008, 2010, 2012, 2014, 2016.

Del mismo modo, ha llegado a ser un lugar común sostener que la confianza en las instituciones políticas está pasando por una crisis, pero en realidad, tenemos escasos estudios que han abordado la confianza en instituciones de manera particular, pues suelen fusionar los distintos tipos de confianza en índices o factores. Ello es positivo, pero también evita y aísla la posibilidad estudiar los efectos de las capacidades institucionales y del apoyo político en municipalidades. Asimismo, no existe estudio en Chile que aborde la problemática de la confianza en las municipalidades desde una perspectiva contextual en niveles distintos a los individuos o en agrupaciones comunales. Las instituciones importan, pero ello debe ser comprobado con técnicas acordes para ello. Dicha brecha de investigación pretende ser llenada con este estudio, controlando por variables del nivel individual que han sido profusamente testeadas con la teoría del apoyo político de Easton.

La pregunta de investigación de este estudio es si las capacidades institucionales de las municipalidades inciden en la confianza política que las personas depositan en ellas y, de existir tal influencia, cómo es dicha relación. El argumento principal es que las municipalidades que presenten un mayor nivel de capacidades institucionales (administrativas-técnicas, políticas y extractivas/fiscal) presentarán mayores niveles de confianza en el municipio por parte de los residentes de tales comunas. Esta afirmación se basa en 
que las personas al ser consultadas por la confianza en los gobiernos locales efectúan evaluaciones a la gestión de los municipios en las comunas que residen. Dichas evaluaciones serán distintas en la medida que se ponderen efectos de interacción entre los indicadores de capacidad institucional, como también por los factores que han sido medidos en la teoría del apoyo político.

En línea con lo anterior, este estudio emplea estadística descriptica, análisis de componentes principales y análisis multinivel. Al ser una investigación que combina distintos niveles de medición (individual y contextual), se recurre a tres bases de datos. La primera es LAPOP para medir el nivel individual y la variable dependiente, en tanto que se recurren a datos electorales (Servicio Electoral [SERVEL] 2018) e información de los municipios del país para el año 2016 (Sistema Nacional de Información Municipal [SINIM] 2018).

El artículo se organiza de la siguiente forma. Primero, se expone una revisión de literatura que abarca los principales conceptos de este estudio, tales como apoyo político; confianza en las instituciones políticas y las capacidades institucionales. Segundo, se presenta la sección de metodología, en la cual se describe la hipótesis, la estrategia cuantitativa (bases de datos usadas, variables contextuales e individuales y su operacionalización), el método utilizado y las limitaciones metodológicas. Tercero, se exponen los resultados, diferenciándolos por hallazgos a nivel contextual y a nivel individual. Finalmente, se presentan las conclusiones del estudio.

\subsection{TEORÍA DEL APOYO POLÍTICO}

Una manera que está ampliamente desarrollada en la ciencia política es considerar a la confianza institucional como una dimensión de la teoría del apoyo político. Esta teoría fue formulada por Easton 1957, 1965, 1975, 1976, la cual ha sido expandadia por otros autores (Listhaug 1995; Anderson y Guillory 1997; Mishler y Rose 1997; Fuchs et al. 1995; Fuchs y Klingemann 1995; Klingemann 1999; Dalton 1999, 2004, 2014; Norris 1999a, 2011, 2017; Putnam et al., 2000; Newton y Norris 2000; Thomassen 1995; Anderson et al. 2005; Criado y Herreros 2007; Booth y Seligson 2009; Luna et al. 2010; Salinas y Booth 2011; Luna et al. 2012).

El modelo de apoyo político de Easton es una propuesta para examinar la democracia representativa, en tanto legitimidad, en la que los ciudadanos actúan utilizando su voto y delegando el poder en las élites políticas. Easton sostiene que el apoyo es: "una actitud por la cual una persona se orienta hacia un objeto ya sea a favor o en contra" (1975:436) dentro de un sistema político. Tal actitud es "energía en forma de acciones u orientacio- 
nes que promueven y resisten un sistema político, las demandas que surgen en él, y las decisiones que surgen de él también deben ser puestas en el sistema para mantenerlo en funcionamiento" (Easton 1957:390). Para Easton $(1957,1965,1975,1976)$, un sistema político se apoya en tres objetos políticos: comunidad política, régimen y autoridades. El apoyo político de los ciudadanos se entiende como una contribución o una exigencia al sistema. Tales insumos son transformados por las élites a través de procesos de formulación de políticas, leyes y decisiones públicas rutinarias. Hay dos tipos de apoyo: específico y difuso. El apoyo específico se refiere a las evaluaciones que los individuos hacen de las autoridades públicas y los resultados del sistema. El apoyo difuso se refiere a la adhesión a un conjunto de valores compartidos que son dominantes en un régimen político en un momento dado.

\subsection{CONFIANZA Y APOYO POLÍTICO}

En 2000, Newton y Norris analizaron las causas a largo plazo y los patrones de confianza en las instituciones de las democracias trilaterales. Para los autores, el deterioro de la confianza institucional es mucho más grave que la disminución de la confianza en los políticos o en determinadas autoridades. De hecho, una "pérdida de confianza en las instituciones puede ser un mejor indicador del descontento público con el mundo moderno porque son los pilares básicos de la sociedad" (Newton y Norris 2000:53). Utilizando la Encuesta Mundial de Valores de 1980-4 y 1990-3 en diecisiete países desarrollados, y desplegando un análisis multivariado a nivel individual, Newton y Norris proponen dos índices para medir el apoyo público a las instituciones como variables dependientes: las instituciones públicas y las instituciones privadas y sin fines de lucro. Los autores encuentran un retroceso de la confianza en las instituciones públicas en casi todos los países estudiados durante la década de 1980. Además, hay oscilaciones destacadas entre los dos índices. Los autores interpretan esto como un efecto del fin de la Guerra Fría, dado que la gente ya no tiene que defender a sus países en términos ideológicos, por tanto, pueden evaluar sus instituciones más críticamente que antes. Este aspecto arroja luz sobre el hecho que el contexto de un país es crucial a la hora de evaluar nuevas hipótesis y teorías.

Para Listhaug, "la confianza política constituye un aspecto importante de las orientaciones democráticas de los públicos de masas" (1995:294). El autor añade que "una disminución de la confianza en las instituciones es potencialmente más grave que un debilitamiento de la confianza política" (Listhaug 1995:294). Listhaug hace dos contribuciones a la teoría del apoyo político: metodológica y empírica. Primero, el investigador introduce una perspectiva multidimensional para medir la confianza política. Segundo, 
introduce la lógica de la brecha entre el ganador y el perdedor, que serían mejoradas más tarde por Anderson y Guillory (1997). Al utilizar estudios sobre elecciones nacionales realizados en Dinamarca, Noruega, Suecia y los Países Bajos de 1968 a 1989, y al aplicar un análisis descriptivo y multivariado a nivel individual, los hallazgos de Listhaug implican que los cambios en la confianza están influenciados por las alternancias en el gobierno, el papel de la incumbencia y el desempeño de los partidos en el gobierno. En este sentido, cómo y para quién votar, y la autoubicación ideológica, son elementales a la hora de evaluar el sistema político. En segundo lugar, la confianza política, la aprobación de autoridades específicas en gobiernos y líderes partidarios son inestables en el tiempo. Estas dimensiones forman parte de una evaluación específica del apoyo, en términos de Easton, y no deberían representar una amenaza para la democracia.

Mishler y Rose (1997) analizaron la estructura y determinantes de la confianza pública en las instituciones políticas de nueve regímenes poscomunistas de Europa Central y Oriental. Este trabajo hace tres contribuciones destacadas a la teoría del apoyo político. Primero, la selección de casos va más allá de la típica muestra general de países trilaterales. Segundo, el contexto histórico en el que se disponía de datos era crucial para evaluar los aspectos críticos de la tercera ola de democratización. Tercero, Mishler y Rose (1997) dan a la teoría del apoyo político un mejor tratamiento de la confianza política, ya que realizaron un análisis factorial que permitió una mejor y más profunda comprensión de la confianza política en las nuevas democracias.

\subsection{CONFIANZA EN LAS INSTITUCIONES PÚBLICAS EN CHILE}

Si bien variados políticos y académicos se refieren a la confianza como una variable crucial para el sistema político, los estudios sobre confianza en las instituciones públicas son más bien escasos en Chile (Morales 2008; Segovia et al. 2008; ICSO 2015). Dicha escasez se acrecienta cuando el objeto de análisis son las municipalidades, salvo excepciones (Centro UC Políticas Públicas 2016a, 2016b; Somma et al. 2016).

Morales (2008) exploró los niveles y determinantes de diferentes tipos de confianza institucional hallados en los ciudadanos chilenos. Utilizando las encuestas LAPOP 2006 y 2008, y empleando un análisis multivariado tipo OLS al nivel de análisis individual, Morales aplicó un análisis de componentes principales para construir índices de confianza en las instituciones políticas (gobierno, parlamento, poder judicial, partidos políticos), y confianza en las Fuerzas Armadas y Carabineros. Cabe decir que Morales 
no incluyó en la variable latente de confianza en las instituciones políticas a las municipalidades. Los principales hallazgos de Morales fueron:

"quienes perciben que el gobierno realiza un esfuerzo por solucionar los temas más importantes para el país, quienes se identifican con la Concertación, y quienes declaran haber votado en la última elección presidencial poseen niveles de confianza en las instituciones democráticas mayores a los de sus conciudadanos. Por otro lado, los más educados, de mayor edad, y aquellos que viven en lugares pequeños, también tienden a confiar más que sus pares en la institucionalidad democrática" (2008:182).

En 2008, Segovia et al. con una postura evaluativa de la confianza en las instituciones políticas, sostienen que la confianza se explicaría por la capacidad (preparación y recursos) de las instituciones políticas para cumplir sus metas, como por los esfuerzos por encauzar tales metas institucionales hacia y en beneficio de las personas (benevolencia). Usando una encuesta aplicada en el 2005 a casi un millar de personas en la capital de Chile, junto a la utilización de regresiones lineales simples a nivel individual, los autores emplearon la pregunta de confianza, como variable dependiente, para once instituciones públicas (carabineros, policía de investigaciones, presidente, municipios, fuerzas armadas, gobierno, ministerio público, congreso, tribunales de justicia, corte suprema, y partidos políticos). Los principales hallazgos de Segovia et al. (2008) fueron que, tanto la capacidad como la benevolencia, resultaron ser fuertes predictores de la confianza en las instituciones públicas. Del mismo modo, la capacidad resultó ser un factor explicativo más robusto que la benevolencia, en tanto que la interacción entre ambas también resultó ser estadísticamente significativa.

Desde una perspectiva descriptiva, el ICSO (2015) efectuó un análisis de tendencias sobre la confianza en instituciones políticas para el periodo 2008-2015. Utilizando encuestas nacionales de opinión pública realizadas por la Universidad Diego Portales, ICSO (2015) midió la confianza en once instituciones (radio, carabineros, fuerzas armadas, diarios, iglesia católica, televisión, gobierno, grandes empresas, tribunales de justicia, congreso, y partidos políticos). Cabe decir que ICSO (2015) no incluyó en la variable latente de confianza en las instituciones políticas a las municipalidades. Los principales hallazgos de ICSO (2015) evidencian que los niveles de confianza decaen para todas las instituciones, que dichas bajas son graduales (salvo la confianza en el poder ejecutivo en el 2010 y la confianza en la iglesia católica en el 2011), y que la confianza en el gobierno es mayor entre quienes evalúan mejor el trabajo conglomerado político gobernante, lo cual es consistente con el trabajo de Anderson y Guillory (1997). 
El Centro UC Políticas Públicas en el año 2016 desarrolló dos estudios en donde analizó, con distinto grado de profundidad y metodologías, la confianza en las municipalidades. El primer estudio, de corte cualitativo (abril 2016), apuntó a explorar y caracterizar los factores que afectan a la confianza en el gobierno local (Centro UC Políticas Públicas 2016a). Este estudio se efectuó en cuatro comunas de la región metropolitana (San Bernardo, El Bosque, Peñalolén, y Providencia). Las técnicas de recolección de información implementadas fueron grupos focales con vecinos no organizados, entrevistas grupales a dirigentes sociales de organizaciones, jornadas de reflexión con trabajadores municipales y doce entrevistas a expertos, alcaldes y administradores municipales (Centro UC Políticas Públicas 2016a). En términos conceptuales, y al igual que Segovia et al. (2008), esta investigación entiende que la confianza es una función evaluativa entre expectativas y realidad. Si bien los hallazgos de este estudio son relevantes y muy nutridos, en esta ocasión se hará una selección con las conclusiones que están línea con la pregunta d investigación de este artículo. Los principales hallazgos fueron que la confianza en la municipalidad se explicaría por una conjugación de elementos de tipo evaluativo: integridad y competencia técnica de los trabajadores municipales y autoridades, como por familiaridad (cercanía e informalidad) y control (capacidad de fiscalizar y sancionar). De este modo, el Centro UC Políticas Públicas sostiene que "el desajuste de la confianza de las expectativas de los vecinos frente a los que esperan del municipio y lo que este hace en la práctica se traduciría en desconfianza" (2016:17).

El concepto de confianza implica riesgos, pero dichos riesgos pueden ser aminorados a través de la familiariadad y del control (Centro UC Políticas Públicas 2016a). Respecto al primero, la interacción frecuente y la proximidad son condiciones necesarias, pero no suficientes, para el cumplimiento de acuerdos y expectativas entre las partes. Por ejemplo, alcaldes incumbentes podrían generar mayores expectativas de cumplimiento de promesas por el hecho de ser conocidos, mantener continuidad en los equipos de gestión y en su estilo de dirección. En este sentido, deberíamos esperar que en comunas donde el alcalde es incumbente, la confianza debería ser mayor que en las comunas donde el alcalde es nuevo. En relación con el control, este mecanismo dice relación a los castigos y deberes institucionales que son definidos por una tercera parte, los cuales regulan la relación entre las partes.

El segundo estudio desarrollado por el Centro UC Políticas Públicas en el año 2016 (septiembre) fue de corte cuantitativo. El objetivo fue realizar un diagnóstico sobre las percepciones, actitudes y comportamientos ciudadanos en un serie de temáticas relevantes en lo local, tales como "conocimiento y evaluación de servicios municipales, confianza, participación, atributos para autoridades y funcionarios municipales, problemas comunales y 
las medidas que las personas esperan que el municipio realice" (Centro UC Políticas Públicas 2016b: 4). Esta encuesta de opinión tuvo un tamaño muestral de 1.516 casos, cubrió 92 comunas y fue aplicada entre junio y julio de 2016. Los principales hallazgos fueron que los municipios presentan mayores niveles de confianza en comparación al sistema político en general, que las personas confían más en los actores que están más cerca a ellos, que las personas mayores creen más en su municipio o alcalde que los jóvenes y adultos, en tanto que el nivel socioeconómico no tiene un patrón lineal explicativo y que el tamaño de la comuna no influye en la percepción de confianza (Centro UC Políticas Públicas 2016b).

\subsection{CAPACIDADES INSTITUCIONALES DE LOS MUNICIPIOS}

Las capacidades institucionales, fundamentalmente las capacidades administrativas y políticas, son entendidas como factores contextuales que influencian las percepciones de los individuos sobre la confianza en las municipalidades. Ambos tipos de capacidades fueron seleccionadas por su prevalencia para las capacidades estatales (Grindle 1996; Repetto 2004; Completa 2017), en tanto condiciones necesarias para fortalecer los recursos institucionales.

La clasificación ideada por Grindle (1996) es la dominante al momento de estudiar las capacidades estatales. Esta autora identifica cuatro dimensiones que son las que promoverían la gobernabilidad y el desarrollo. Estas dimensiones son: institucional, técnica, administrativa y política.

En referencia a la capacidad institucional, ésta apunta a regular los campos de la economía y la política en una sociedad, constriñendo comportamientos a través de la exigencia del cumplimiento de las normas formales.

Referente a la capacidad técnica, ésta apunta a diseñar, implementar y evaluar las políticas, planes y programas públicos.

En relación con la capacidad administrativa, ésta se distingue por la presencia de dos tipos de recursos claves: personal calificado para cumplir con la función pública y recursos financieros para solventar las acciones estatales. Estos tipos de recursos son los que permitirían desarrollar las funciones y atribuciones esenciales de los gobiernos sub-nacionales. Cabe precisar que la línea divisoria entre la capacidad técnica y administrativa podría tornarse difusa en ciertos momentos. Para zanjar dicha disyuntiva, este estudio las fusiona para así simplificar la interpretación de esas dimensiones. En tanto, la capacidad política se asocia con mecanismos y formas legítimas 
de resolución de controversias generadas por los problemas sociales y las demandas ciudadanas. Para canalizar los conflictos, se necesitan líderes, autoridades y directivos que respondan de manera efectiva a las demandas y participación ciudadana (Rodríguez-Gustá 2004), movilizando los recursos necesarios para dar cumplimento a los objetivos institucionales (Freigedo et al. 2015).

Finalmente, y considerando los aportes de Giglio (2016) referentes a la identificación de una capacidad extractiva/fiscal de los estados, se agrega una quinta dimensión a las cuatro identificadas por Grindle (1996). Sin embargo, también se podría argumentar que la propuesta de Giglio (2016) es una diferenciación de la capacidad administrativa señalada anteriormente. Dicha distinción respondería a las fases del financiamiento. Por un lado, está: "la capacidad fiscal y extractiva que [el Estado] posee para la adquisición de recursos y bienes. [De esta forma], es necesario tener en cuenta la normativa y legislación existente que define los alcances de dicha capacidad en los diferentes niveles de Gobierno y cuáles son las competencias de cada uno de ellos en la materia" (Giglio 2016: 249-250).

Por el otro lado, está la disponibilidad de los recursos financieros y presupuestarios que son parte de la capacidad administrativa y técnica para cumplir con la función pública.

\section{MET O D O L O G Í A}

Atendiendo a la pregunta de investigación señalada más arriba, este artículo desarrolla su análisis en dos niveles de medición. Primero, el nivel individual que es capturado por la encuesta LAPOP 2016. Ésta es usada para medir el impacto de distintas variables y modelos. Estos modelos son: factores demográficos; factores políticos e ideológicos; capital social; cambio valórico; consumo de medios de comunicación; victimización, inseguridad y corrupción (Booth y Seligson 2009; Fuentes-González 2018).

Respecto al nivel contextual, el supuesto que lo sustenta en esta investigación es que el contexto local importa al momento de las percepciones ciudadanas. Los seres humanos habitan hogares que se encuentran en barrios, que a su vez se encuentran en comunas, regiones, países, etc. Estos grupos tienen diferentes contextos, que a menudo se pasan por alto cuando se explican las actitudes individuales. El beneficio de la duda al menos nos dice que los contextos políticos, legales, culturales, históricos y socioeconómicos podrían tener un impacto en la vida de las personas. Por tanto, se espe- 
ra que los factores contextuales también influyan en la toma de decisiones. Estas características hacen que los datos recogidos en las encuestas tengan una agrupación implícita, es decir, que exista una estructura jerárquica en la recogida de datos (Goldstein y Silver 1989; Paterson y Golstein 1991; Goldstein 1995; Snijders y Bosker 1999; Rasbash 2008; Acik-Toprak et al. 2009; Bühlmann 2012). Para efectos de esta investigación, dicha agrupación jerárquica corresponde a las cien comunas de la encuesta LAPOP (2016).

\subsection{HIPÓTESIS}

El argumento de este estudio es que las municipalidades que presenten un mayor nivel de capacidades institucionales (administrativas-técnicas, políticas y extractivas/fiscal) presentarán mayores niveles de confianza en el gobierno local por parte de los residentes de tales comunas. Esta afirmación se basa en que las personas al ser consultadas por la confianza en los municipios efectúan evaluaciones a la gestión de éstos en las comunas que residen. Dichas evaluaciones serán distintas en la medida que se ponderen efectos de interacción entre los indicadores de capacidad institucional, como también por los factores que han sido medidos en la teoría del apoyo político mencionados en la sección anterior.

De acuerdo con lo que se ha argumentado hasta ahora, en este estudio se testean dos hipótesis:

H1: A mayor nivel de capacidades institucionales a nivel municipal, se espera que la percepción individual de confianza en las municipalidades aumente.

Las dimensiones de capacidad institucional a testear son:

-Tasa de colocación laboral [capacidad administrativa-técnica].

-Promedio de profesionales (planta y contrata) del municipio [capacidad administrativa-técnica].

-Ingresos generados por impuestos/derechos/permisos locales [capacidad extractiva/fiscal].

-Periodos en el cargo de alcalde de la elección octubre 2016 [capacidad política].

-Tamaño del concejo municipal [capacidad política].

-Índice institucional para el gobierno abierto municipal IIGAM Chile 2016 [capacidad política].

H2: La asociación entre las capacidades administrativas-técnicas y la confianza en la municipalidad variará, dependiendo de la capacidad política que se esté midiendo. Es decir, el efecto de las capacidades administrati- 
vas-técnicas sobre la confianza en la municipalidad dependerá del tipo de capacidad políticas a nivel comunal en Chile que se esté midiendo.

Sumado a lo anterior, el testeo contemplado en el análisis multinivel es controlado por una serie de variables provenientes de la teoría de apoyo político, con la finalidad de robustecer el análisis y así mitigar, en la medida de lo posible, sesgos de medición.

\subsection{ESTRATEGIA CUANTITATIVA}

\section{a)Instrumentos de medición:}

-LAPOP: Es el instrumento para medir los indicadores desde el nivel individual es LAPOP 2016. LAPOP es una encuesta de opinión pública y comportamiento democrático que cubre las Américas (Norte, Centro, Sur y el Caribe), realizada por la Universidad de Vanderbilt. LAPOP utiliza muestras probabilísticas nacionales de adultos en edad de votar. Para esta investigación se utiliza la última encuesta disponible, correspondiente a 2016-17.

-Las autoridades locales elegidas de 1992 a 2016: SERVEL administra la información electoral en Chile. La información analizada corresponde a siete elecciones locales: 1992, 1996, 2000, 2004, 2008, 2012 y 2016. Esta información electoral entrega datos para las variables contextuales de capacidad política.

-SINIM: Es un sistema de información de cobertura nacional, para los 345 municipios, que recopila, ordena, procesa y pone a disposición pública información dispersa del ámbito local-municipal para distintas áreas. SINIM entrega datos para las variables contextuales de capacidad administrativa-técnica y capacidad extractiva/fisca.

b) Variable dependiente

\section{TABLA 1: DEFINICIÓN, OPERACIONALIZACIÓN, FUENTES DE LA VARIABLE DEPENDIENTE}

\begin{tabular}{|l|l|}
\hline \multicolumn{1}{|c|}{ Variable dependiente } & \multicolumn{1}{c|}{ Definición, operacionalización y fuentes } \\
\hline $\begin{array}{l}\text { Confianza en la } \\
\text { municipalidad }\end{array}$ & $\begin{array}{l}\text { D: Confianza en la municipalidad. } \\
\text { O: Escala que mide la confianza en las municipalidades, yendo de } \\
1 \text { (nada de confianza) hasta } 7 \text { (mucha confianza), pregunta B32. } \\
\text { Variable escalar. } \\
\text { F: LAPOP 2016. }\end{array}$ \\
\hline
\end{tabular}

FUENTE: ELABORACIÓN PROPIA CON DATOS DE LAPOP 2016. 
TABLA 2: DEFINICIÓN, OPERACIONALIZACIÓN, FUENTES DE LAS VARIABLES

\begin{tabular}{|c|c|c|}
\hline $\begin{array}{c}\text { Variable } \\
\text { independiente }\end{array}$ & Definición, operacionalización y fuentes & $\begin{array}{l}\text { Dirección } \\
\text { esperada }\end{array}$ \\
\hline $\begin{array}{l}\text { Porcentaje de } \\
\text { colocación } \\
\text { laboral }\end{array}$ & $\begin{array}{l}\text { D: Porcentaje de colocación laboral. } \\
\text { O: (Personas Enviadas a un Empleo / Personas Inscritas en la } \\
\text { Municipalidad en Busca de Empleo) * 100. Variable escalar. } \\
\text { F: SINIM } 2018 .\end{array}$ & + \\
\hline $\begin{array}{l}\text { Porcentaje de } \\
\text { profesionales } \\
\text { (planta y } \\
\text { contrata) del } \\
\text { municipio }\end{array}$ & $\begin{array}{l}\text { D: Porcentaje de profesionales del municipio } \\
\text { 0: (Número de profesionales de planta + Número de } \\
\text { profesionales a contrata) / (número total de funcionarios } \\
\text { de planta + número total de funcionarios a contrata) * } 100 . \\
\text { Variable escalar. } \\
\text { F: SINIM } 2018 .\end{array}$ & + \\
\hline $\begin{array}{l}\text { Ingresos } \\
\text { generados por } \\
\text { impuestos/ } \\
\text { derechos/ } \\
\text { permisos locales }\end{array}$ & $\begin{array}{l}\text { D: Ingresos propios permanentes. } \\
\text { O: Log natural (derechos de aseo + derechos varios + multas } \\
\text { e intereses + part. impuesto territorial + patentes municipales } \\
\text { a beneficio municipal + permiso de circulación a beneficio } \\
\text { municipal + renta de inversiones). Variable escalar. } \\
\text { F: SINIM } 2018 .\end{array}$ & + \\
\hline $\begin{array}{l}\text { Periodos en el } \\
\text { cargo de alcalde } \\
\text { de la elección } \\
\text { octubre } 2016\end{array}$ & $\begin{array}{l}\text { D: Periodos de incumbencia alcalde actual } \\
\text { O: Número de veces consecutivas que el alcalde actual ha sido } \\
\text { electo alcalde en la misma comuna. Variable escalar. } \\
\text { F: SERVEL } 2018 .\end{array}$ & + \\
\hline $\begin{array}{l}\text { Tamaño } \\
\text { del concejo } \\
\text { municipal }\end{array}$ & $\begin{array}{l}\text { D: Número de concejales por municipio. } \\
\text { 0: } 6 \text { concejales en comunas de hasta } 70 \text { mil electores; } 8 \\
\text { concejales en comunas de más de } 70 \text { mil electores y hasta } 150 \\
\text { mil electores; } 10 \text { Diez concejales en comunas de más de } 150 \\
\text { mil electores. Variable escalar. } \\
\text { F: SERVEL } 2018 .\end{array}$ & + \\
\hline $\begin{array}{l}\text { Índice institucional } \\
\text { para el gobierno } \\
\text { abierto municipal - } \\
\text { IIGAM Chile } 2016\end{array}$ & $\begin{array}{l}\text { D: Herramienta de diagnóstico y línea de base sobre la } \\
\text { implementación del gobierno abierto en las municipalidades } \\
\text { chilenas. } \\
\text { O: Índice ponderado de cuatro dimensiones (Transparencia } \\
\text { Activa 22\%; Acceso a la información } 22 \% \text {; Participación } \\
\text { ciudadana 22\%; Iniciativas propias 34\%). Variable escalar. } \\
\text { F: Hernández } 2016 \text {. }\end{array}$ & + \\
\hline
\end{tabular}

FUENTE: ELABORACIÓN PROPIA EN BASE A PRIBBLE 2017, CON DATOS DE SINIM 2018; SERVEL 2018; HERNÁNDEZ 2016. 
d) Variables independientes a nivel individual

TABLA 3: DEFINICIÓN, OPERACIONALIZACIÓN, FUENTES DE LAS VARIABLES

\begin{tabular}{|c|c|c|}
\hline Variable & Definición, operacionalización y fuentes & $\begin{array}{l}\text { Dirección } \\
\text { esperada }\end{array}$ \\
\hline Género & $\begin{array}{l}\text { D: Género del entrevistado. } \\
\text { 0: Categorías originales de la pregunta Q1 se recodifican, siendo } \\
\text { (1) Hombre (0) Mujer. Variable nominal. } \\
\text { F: LAPOP } 2016 .\end{array}$ & $\begin{array}{l}\text { Sin } \\
\text { efecto }\end{array}$ \\
\hline Edad & $\begin{array}{l}\text { D: Edad. } \\
\text { O: Q2. = ¿Cuál es su edad en años cumplidos? Variable escalar. } \\
\text { F: LAPOP } 2016 .\end{array}$ & + \\
\hline $\begin{array}{l}\text { Nivel de } \\
\text { escolaridad }\end{array}$ & $\begin{array}{l}\text { D: Años de escolaridad. } \\
\text { O: ED. = ¿Cuál fue el último año de educación que usted } \\
\text { completó o aprobó? Variable escalar. } \\
\text { F: LAPOP } 2016 .\end{array}$ & - \\
\hline $\begin{array}{l}\text { Ingreso del } \\
\text { hogar }\end{array}$ & $\begin{array}{l}\text { D: Es un índice sumativo del ingreso del hogar. } \\
\text { O: Suma de las preguntas R1, R3, R4, R4A, R5, R6, R7, R8, R12, } \\
\text { R14, R15, R16, R18, R26, formando un índice de } 0 \text { a 16. Variable } \\
\text { escalar. } \\
\text { F: LAPOP 2016; Fuentes-González } 2018 .\end{array}$ & + \\
\hline $\begin{array}{l}\text { Localización } \\
\text { Urbano/Rural }\end{array}$ & $\begin{array}{l}\text { D: Localización Urbano/Rural. } \\
\text { O: Categorías originales de la pregunta UR se recodifican, siendo } \\
\text { (1) Urbano (0) Rural. Variable nominal. } \\
\text { F: LAPOP } 2016 .\end{array}$ & + \\
\hline $\begin{array}{l}\text { Brecha ganador- } \\
\text { perdedor } \\
\text { (elecciones } \\
\text { presidenciales) }\end{array}$ & $\begin{array}{l}\text { D: Brecha ganador-perdedor. } \\
\text { O: La pregunta es VB3N. El ganador de la categoría es (1), } \\
\text { mientras que el resto de las categorías se agrupan en (0). } \\
\text { Cabe señalar que la categoría (0) tiene en cuenta: el candidato } \\
\text { derrotado, ninguno (voto en blanco o anulado), otro, no sabe, no } \\
\text { contesta, y una persona que no votó. Variable nominal } \\
\text { F: LAPOP 2016; Fuentes-González } 2018 .\end{array}$ & - \\
\hline
\end{tabular}




\begin{tabular}{|c|c|c|}
\hline Variable & Definición, operacionalización y fuentes & $\begin{array}{l}\text { Dirección } \\
\text { esperada }\end{array}$ \\
\hline $\begin{array}{l}\text { Orientación } \\
\text { ideológica }\end{array}$ & $\begin{array}{l}\text { D: Orientación ideológica. } \\
\text { 0: Las categorías, de la pregunta L1, se agregaron de la } \\
\text { siguiente manera: } \\
\text { [1-3] = Izquierda; [4-7] = Centro [8-10] = Derecha; }[88,98] \text { = Sin } \\
\text { identificación (categoría de referencia). Variable nominal. } \\
\text { F: LAPOP 2016; Dalton 2006: } 293 \text {. }\end{array}$ & - \\
\hline Rol del Estado & $\begin{array}{l}\text { D: Índice sumativo del rol del Estado. } \\
\text { O: Suma las preguntas ROS1 (Estado empresario) y ROS4 } \\
\text { (Estado lucha contra la desigualdad), formando un índice de } 0 \text { a } \\
\text { 14. Variable escalar. } \\
\text { F: LAPOP } 2016 .\end{array}$ & - \\
\hline $\begin{array}{l}\text { Participación en } \\
\text { organizaciones } \\
\text { sociales }\end{array}$ & $\begin{array}{l}\text { D: Índice sumativo de participación en organizaciones sociales. } \\
\text { 0: Primero, se cambió el orden las categorías de las preguntas } \\
\text { CP6 (organización religiosa), CP7 (reuniones de escolares), CP8 } \\
\text { (reuniones comunitarias), CP13 (partido/movimiento político). } \\
\text { Segundo, se agrupan las cuatro preguntas, formando un índice } \\
\text { de } 0 \text { a 14. Variable escalar. } \\
\text { F: LAPOP } 2016 \text {. }\end{array}$ & $\begin{array}{l}\text { Sin } \\
\text { efecto }\end{array}$ \\
\hline $\begin{array}{l}\text { Confianza en } \\
\text { miembros de la } \\
\text { comunidad }\end{array}$ & $\begin{array}{l}\text { D: Escala de confianza en miembros de la comunidad } \\
\text { 0: Primero, se cambió el orden las categorías de la pregunta IT1. } \\
\text { Segundo, se formula escala de } 1 \text { a 4, donde } 1 \text { representa nada } \\
\text { confiable y } 4 \text { muy confiable. Variable escalar. } \\
\text { F: LAPOP } 2016 .\end{array}$ & + \\
\hline $\begin{array}{l}\text { Confianza en } \\
\text { las instituciones } \\
\text { públicas }\end{array}$ & $\begin{array}{l}\text { D: Índice de confianza en las instituciones públicas. } \\
\text { 0: Análisis de componentes principales, preguntas = b12 b13 } \\
\text { b18 b21 b21a b37 b47a } \\
\text { F: LAPOP } 2016 \text {. }\end{array}$ & + \\
\hline Cambio valórico & $\begin{array}{l}\text { D: Índice sumativo del cambio valórico. } \\
\text { O: Suma las preguntas D5 (postulación de homosexuales a } \\
\text { cargos públicos) y D6 (matrimonio homoparental), formando un } \\
\text { índice de } 0 \text { a 20. Variable escalar. } \\
\text { F: LAPOP } 2016 .\end{array}$ & $\begin{array}{l}\text { Sin } \\
\text { efecto }\end{array}$ \\
\hline
\end{tabular}




\begin{tabular}{|c|c|c|}
\hline Variable & Definición, operacionalización y fuentes & $\begin{array}{l}\text { Dirección } \\
\text { esperada }\end{array}$ \\
\hline $\begin{array}{l}\text { Consumo de } \\
\text { medios de } \\
\text { comunicación }\end{array}$ & $\begin{array}{l}\text { D: Nivel de consumo de medios de comunicación. } \\
\text { O: Primero, se cambió el orden las categorías de la pregunta } \\
\text { GIO. Segundo, se formula escala de } 1 \text { a 5, donde } 1 \text { representa } \\
\text { nunca y } 5 \text { diariamente. Variable escalar. } \\
\text { F: LAPOP } 2016 .\end{array}$ & - \\
\hline Victimización & $\begin{array}{l}\text { D: Victimización. } \\
\text { O: Categorías originales de la pregunta VIC1EXT se recodifican, } \\
\text { siendo (1) Sí (0) No. Variable nominal. } \\
\text { F: LAPOP } 2016 .\end{array}$ & $\begin{array}{l}\text { Sin } \\
\text { efecto }\end{array}$ \\
\hline $\begin{array}{l}\text { Percepción de } \\
\text { seguridad }\end{array}$ & $\begin{array}{l}\text { D: Percepción de seguridad. } \\
\text { O: Primero, se cambió el orden las categorías de la pregunta } \\
\text { A0J11. Segundo, se formula escala de } 1 \text { a } 5 \text {, donde } 1 \text { significa } \\
\text { muy inseguro y } 5 \text { muy seguro. Variable escalar. } \\
\text { F: LAPOP } 2016 \text {. }\end{array}$ & + \\
\hline $\begin{array}{l}\text { Percepción de } \\
\text { corrupción en } \\
\text { los políticos }\end{array}$ & $\begin{array}{l}\text { D: Percepción de corrupción en los políticos } \\
\text { O: EXC7NEW. = Pensando en los políticos de Chile, ¿cuántos } \\
\text { de ellos cree usted que están involucrados en corrupción? (1) } \\
\text { Ninguno (2) Menos de la mitad (3) La mitad de los políticos (4) } \\
\text { Más de la mitad (5) Todos. Variable escalar. } \\
\text { F: LAPOP } 2016 .\end{array}$ & - \\
\hline $\begin{array}{l}\text { Preferencia por } \\
\text { la democracia }\end{array}$ & $\begin{array}{l}\text { D: Preferencia por la democracia } \\
\text { O: ING4. = Cambiando de nuevo el tema, puede que la } \\
\text { democracia tenga problemas, pero es mejor que cualquier otra } \\
\text { forma de gobierno. ¿Hasta qué punto está de acuerdo o en } \\
\text { desacuerdo con esta frase? Variable escalar. } \\
\text { F: LAPOP } 2016 .\end{array}$ & + \\
\hline $\begin{array}{l}\text { Satisfacción con la } \\
\text { democracia }\end{array}$ & $\begin{array}{l}\text { D: Satisfacción con la democracia } \\
\text { O: Primero, se cambió el orden las categorías de la pregunta } \\
\text { PN4. Variable escalar. } \\
\text { F: LAPOP } 2016 .\end{array}$ & + \\
\hline
\end{tabular}


e) Método

El tipo de estudio es cuantitativo y las técnicas usadas son: estadística descriptiva y análisis multinivel. La primera técnica busca mostrar tendencias para observar continuidades y discontinuidades de factores, mientras que el segundo apunta a evidenciar si el contexto en el cual están insertas las personas (comuna) afecta o no su percepción respecto a la confianza en la municipalidad.

Dadas las características de las hipótesis que guían este estudio, un análisis multinivel es apropiado por las siguientes cuatro razones. Primero, el contexto en el que vive la gente es importante. Segundo, el método multinivel permite tratar las unidades estudiadas como heterogéneas (Paterson y Goldstein 1991). Este aspecto permite un mayor análisis de varianza y las conceptualizaciones tienden a evitar la homogeneidad de las unidades de análisis. Tercero, el análisis multinivel proporciona estimaciones más robustas que los análisis basados en regresiones OLS o a un solo nivel, lo que nos permite observar las diferencias entre y dentro de los grupos (Paterson y Goldstein 1991; Snijders y Bosker 1999; Hox 2002; Norris 2011). Cuarto, es importante saber si los conjuntos de datos disponibles a nivel individual y comunal contienen suficientes unidades de análisis. Para cumplir con esto, debemos definir los factores necesarios para considerar apropiado el tamaño de la muestra. Utilizando simulaciones de Monte Carlo, Clarke concluye: “Los modelos multinivel pueden estimarse de manera confiable con un promedio de sólo cinco observaciones por grupo" (2007:752). Rasbash (2008) sostiene que un análisis multinivel no requiere un número igual de unidades de micro-nivel en cada unidad de macro-nivel. Además, en el caso de dos niveles, Goldstein y Silver (1989) sugieren que el umbral necesario para que los grupos realicen un análisis multinivel debe establecerse en veinticinco casos. La Tabla 4 expone el cumplimiento de los criterios para el nivel individual y el nivel contextual. Finalmente, el software utilizado para ejecutar análisis multinivel es MLwiN versión 3.02. 
TABLA 4: NÚMERO DE CASOS POR COMUNAS

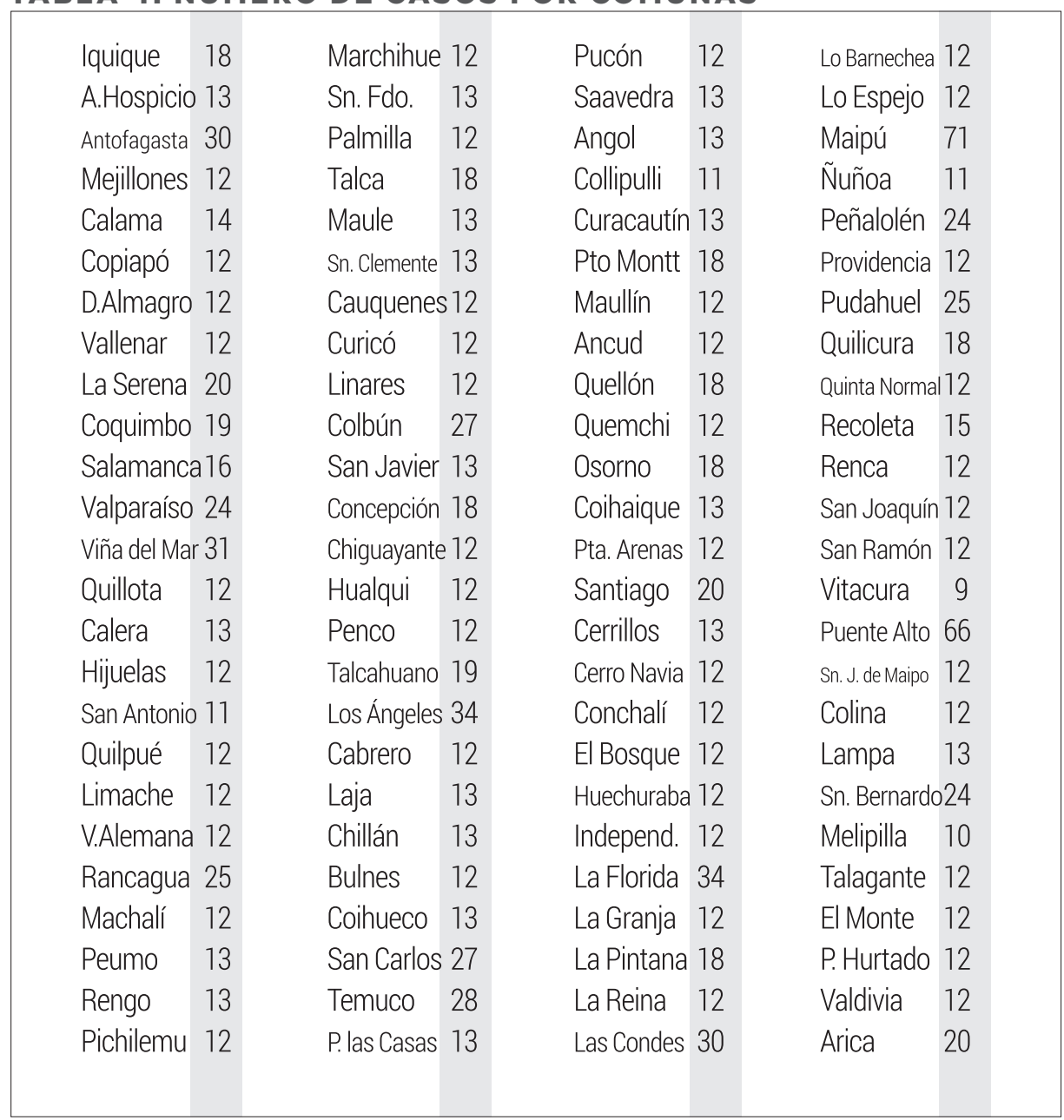

FUENTE: ELABORACIÓN PROPIA CON DATOS DE LAPOP (2016).

\subsection{LIMITACIONES METODOLÓGICAS}

Toda investigación científica tiene limitaciones, sobre todo las relacionadas a la metodología. Este estudio no es la excepción. Primero, se podría haber contemplado más encuestas LAPOP y así haber logrado un análisis longitudinal. Sin embargo, se privilegió contar con la última encuesta LAPOP disponible para así medir el efecto de la incumbencia en un periodo mayor de años. Segundo, algunos casos están perdidos tanto a nivel individual 
como contextual. Para el nivel individual, será necesario explorar técnicas de imputación. Para el nivel contextual, hubo variables, como la tasa de colocación laboral de las comunas de Diego de Almagro y Melipilla que no fueron reportadas a la SUBDERE. Una forma de solucionar este obstáculo es generar promedios de reportes de años cercanos o imputaciones por similitud. Tercero, dado que la encuesta LAPOP está diseñada con una finalidad comparativa de países latinoamericanos, es probable que este instrumento esté mejor preparado para captar efectos sobre temas y autoridades nacionales. Esta limitación se fundamenta en un análisis de regresión multivariado que comparó la confianza en los municipios, confianza en el presidente y confianza en el congreso nacional (como variables dependientes), controladas con las variables de nivel individual contempladas en la Tabla 3 de este artículo. Al cotejar los r cuadrado ajustado de cada modelo, éste estadístico para el modelo de confianza en el gobierno local fue más pobre que las otras dos confianzas institucionales. Una forma de soslayar esto, sería trabajar con un instrumento diseñado sólo para capturar efectos a nivel comunal.

\section{RES U LT A D O S}

\subsection{HALLAZGOS A NIVEL CONTEXTUAL}

Esta sección entrega la evidencia para responder a la pregunta de investigación que guía este estudio y las hipótesis. Si bien nueve modelos fueron testeados, para efectos prácticos sólo se presentan tres modelos. El modelo 1 presenta los efectos directos para el porcentaje de colocación laboral y periodos del alcalde a nivel contextual, junto al efecto de interacción entre ambas variables. En el Modelo 2 se exponen los efectos directos del porcentaje de colocación laboral y número de concejales, junto al efecto de interacción entre ambos factores. En el Modelo 3 se presentan los efectos directos de los ingresos propios permanentes y número de concejales, junto al efecto de interacción entre ambos factores. Para los tres modelos se presentan las variables independientes al nivel individual.

Segundo, cada una de las tres columnas de los modelos presenta los t-ratio. Esta puntuación se forma dividiendo una estimación en cada error estándar. El t-ratio destaca si existe una asociación significativa entre la confianza en la municipalidad y variables independientes, tanto a nivel individual como contextual. A medida que la relación $t$ aumenta en valor absoluto, el impacto de los factores independientes sobre la variable dependiente es mayor, manteniendo constantes otras variables. Tercero, el valor p aceptable en este trabajo sigue el consenso de la mayoría de los estudios en cien- 
cias sociales; 0,05. Cuarto, la Tabla 5 muestra en verde con números blancos las asociaciones significativas y positivas en el nivel de confianza del $95 \%$. Las celdas en rojo con números blancos muestran las relaciones negativas significativas en el nivel de confianza del 95\%. Si bien en el punto tres de este párrafo se mencionan el umbral de significancia del 95\%, las celdas en naranjo con números negros muestran relaciones positivas y negativas significativas en el nivel de confianza del $90 \%$.

En la Tabla 5 se observa que las variables contextuales de capacidades institucionales tienen efectos sobre la confianza en las municipalidades. Sin embargo, no todas las variables de capacidad institucional afectan de la misma manera a la confianza. De hecho, la variable de capacidad política "número de concejales" resultó ser la variable de mayor capacidad explicativa a nivel contextual. Esto se contrapone con la otra variable de capacidad política, los periodos del alcalde actual a la cabeza del municipio, la cual sólo fue significativa al nivel de confianza del $90 \%$.

Siguiendo con el número de concejales, su nivel de explicación se da tanto a nivel de efectos directos como de efectos de interacción. Así, destaca la relación negativa y estadísticamente significativa entre el número de concejales y la confianza en la municipalidad. Es decir, a medida que los Concejos Municipales son más pequeños, la confianza en la municipalidad aumenta en dichas comunas. Este hallazgo está en línea con lo señalado por el Centro UC Políticas Públicas (2016a) en lo referente a la familiaridad y cercanía, pues las comunas con menor número de electores son las que tienen menor número de concejales. Es decir, la confianza en las municipalidades podría tener un mayor efecto en donde las comunas son más pequeñas en tanto número de personas, lo cual podría facilitar el contacto y conocimiento de los concejales. Esto es probable que no se replique fácilmente en comunas más grandes.

Sobre los efectos de interacción, llama la atención que el número de concejales ejerce un efecto moderador tanto con el porcentaje de colocación laboral como con los ingresos propios permanentes. Dichos efectos son similares, pues el número de concejales ejerce un efecto moderador positivo tanto en el porcentaje de colocación laboral como en los ingresos propios permanentes. Estos hallazgos sugieren que la capacidad política tiene un efecto moderador sobre las capacidades administrativas-técnicas y con las capacidades extractivas fiscales. Este resultado refuerza lo sostenido por MacKuen y Brown (1987), Anderson y Guillory (1997), y Norris (2011), referente a que las variables políticas contextuales son relevantes para explicar las percepciones y actitudes a nivel individual. 
Respecto a los efectos directos de las capacidades administrativas-técnicas y extractiva/fiscal, los ingresos propios permanentes tienen un efecto negativo y estadísticamente significativo sobre la confianza en el municipio. Esto podría estar mostrando que, en municipios con menor capacidad para recaudar ingresos por patentes, derechos o impuestos, los individuos confían más en la municipalidad. Esto podría parecer contraintuitivo pues los ciudadanos que viven en comunas con mayor cantidad de recursos financieros podrían confiar más en su gobierno local por el simple hecho que tiene más dinero para invertir en bienes y servicios a ser provistos para la comunidad. A la luz de estos hallazgos, esta última relación pareciera ser negativa. En referencia a el porcentaje de colocación laboral, este indicador presenta el mismo comportamiento que los ingresos propios permanentes, es decir, tiene un impacto adverso sobre la confianza en la municipalidad al nivel individual. Este último hallazgo sugiere que en comunas donde la colocación laboral es menor, la confianza en el municipio aumenta. Al igual que con el indicador de capacidad administrativa-técnica, dicho resultado parece ser contraintuitivo, pues uno esperaría que las personas confíen más en su municipio cuando logran ubicar a más personas en nuevos trabajos. Cabe decir que la variable que mide capacidad administrativa-técnica, porcentaje de profesionales (planta y contrata) del municipio, y la variable que mide capacidad política, IIGAM Chile 2016, no tuvieron significancia estadística. Una posible razón para explicar lo relacionado con el factor de capacidad administrativa-técnica, es que el indicador de porcentaje de profesionales (planta y contrata) del municipio no contempla a los profesionales a honorarios. Dicho dato no está capturado por SINIM (2018). De acuerdo a datos de SINIM (2018), la dotación de personal a planta, contrata y honorarios es de 249 personas en los municipios, en promedio, de las cuales el $44 \%$ corresponde a personas contratadas a honorarios, para el año 2016, en promedio. Del mismo modo, Guarda (2017) sostiene que personal a honorarios en municipios se duplicó entre 2006 y 2016. En consecuencia, el dato que busca medir profesionalización en los municipios está subrepresentado, su medición es espuria y podría estar entregando un falso-positivo. Con relación al IIGAM 2016, y al testear por separado sus dimensiones, es posible afirmar que la dimensión de acceso a la información fue significativa al nivel de confianza del 90\%, mientras que las otras dimensiones no fueron estadísticamente significativas. Intuitivamente, afloran dos posibles explicaciones. La primera es que el indicador está mal formulado metodológicamente, mientras que el segundo punto es que este indicador podría estar sesgado, pues habría que preguntarse qué proporción de residentes de las comunas utilizan transparencia activa y pasiva. Finalmente, cabe agregar que para medir capacidad administrativa-técnica se pensó en usar los datos de SINIM (2018) sobre ingresos por concepto de postulación al 
Fondo Nacional de Desarrollo a educación y salud. Sin embargo, dichos datos muestran que los municipios que generan mayor cantidad de ingresos propios permanentes no obtienen dichos fondos, lo cual no es porque no posean equipos técnicos para postular, sino que no lo hacen porque no son necesarios para su gestión, probablemente.

\subsection{HALLAZGOS A NIVEL INDIVIDUAL}

De los siete modelos que buscan explicar la confianza en los municipios a nivel individual, al sumar sus t-ratios en valores absolutos y dividirlos por el número de combinaciones posibles, se puede apreciar que el modelo de capital social es, por lejos, el conjunto de variables con mayor peso explicativo para la confianza en el gobierno local. Ambas las variables que miden confianza, interpersonal como en las instituciones, son estadísticamente significativas y positivas. A mayor confianza en los miembros de la comunidad y a mayor confianza en las instituciones, mayor es la confianza en la municipalidad. Asimismo, la variable "confianza en las instituciones" es la que posee el t-ratio más alto. Se podría argumentar una multicolinealidad entre esta variable y la variable dependiente, pero una correlación bivariada arrojó el valor de 0,516 al nivel de confianza de 99\%, por tanto, no es una relación perfecta. La participación en organizaciones sociales no tuvo significancia estadística en los tres modelos, lo cual podría decir que la confianza en la municipalidad está ampliamente distribuida, independientemente de la participación en organizaciones sociales a nivel individual. Este hallazgo es consistente con Fuentes-González (2018).

El segundo modelo con mayor peso explicativo es el de legitimidad democrática. Sin embargo, de las dos variables que componen este modelo, sólo la satisfacción con la democracia resultó ser estadísticamente significativa y positiva en los tres modelos, en tanto que la preferencia por la democracia no mostró significancia estadística. Esta disparidad de resultados podría mostrar que, en términos evaluativos, la relación entre satisfacción con la democracia y la confianza en la municipalidad es real, mientras que una apreciación normativa de la democracia no implica una asociación con la confianza en el gobierno local.

El tercer modelo con mayor peso explicativo fue el de victimización, seguridad y corrupción. Tanto la percepción de seguridad como la percepción de corrupción en los políticos fueron variables estables, robustas, positivas y estadísticamente significativas en los tres modelos. A mayor percepción de seguridad y corrupción en los políticos, la confianza en la municipalidad 
aumenta. Sobre estos resultados, hay que precisar dos aspectos. Primero, las dos variables son percepciones y no preguntas sobre hechos concretos, como es el caso de la victimización, cuya variable no tuvo significación estadística en los tres modelos. Segundo, es llamativo que, a mayor percepción de corrupción en los políticos, la confianza en la municipalidad aumente. Intuitivamente, la relación debería ser inversa. De esta forma, faltan estudios cualitativos que profundicen en esta relación.

El cuarto modelo es el cambio valórico. Si bien las tres combinaciones tienen signo positivo, sólo en el tercer modelo este índice tuvo significancia estadística. Tomando en consideración los hallazgos de Fuentes-González (2018), al parecer este tipo de preguntas tiene mayor relación con preguntas de tipo normativo más que evaluativo. Por esta razón, probablemente, no se aprecie una significación estadística mayor.

El quinto modelo es el de consumo de medios de comunicación. Este fue el único modelo sin significancias estadísticas, lo que implica que la exposición o consumo de noticias no reportan un efecto en la confianza municipal.

El sexto modelo con mayor capacidad explicativa fue el político e ideológico. A diferencia de los hallazgos de Fuentes-González sobre legitimidad democrática en Chile (2008-2014), en donde el modelo más fuerte y robusto fue el político e ideológico, en esta ocasión este esquema explicativo resultó ser pobre. La única variable que resultó negativa y estadísticamente significativa fue las personas que se identifican con la izquierda en el eje ideológico, quienes confían menos en la municipalidad en comparación a las personas sin identificación. Otro resultado llamativo es que la brecha ganador-perdedor no tuvo significancia estadística, siendo que en Fuentes-González (2018), Norris (2011) y Anderson Guillory (1997), esta variable resultó ser preponderante para explicar el apoyo político, satisfacción con la democracia y déficit democrático. Las restantes variables centro, derecha, y rol del Estado no tuvieron una significación estadística.

El modelo de factores demográficos resultó ser el más débil de todos los modelos testeados. De las quince combinaciones posibles, sólo años de escolaridad tuvo una significancia estadística negativa. 
TABLA 5: RESUMEN DE LAS ESTIMACIONES ESTADÍSTICAS DE CAPACIDADES INSTITUCIONALES SOBRE LA CONFIANZA EN LA MUNICIPALIDAD, CHILE, 2016

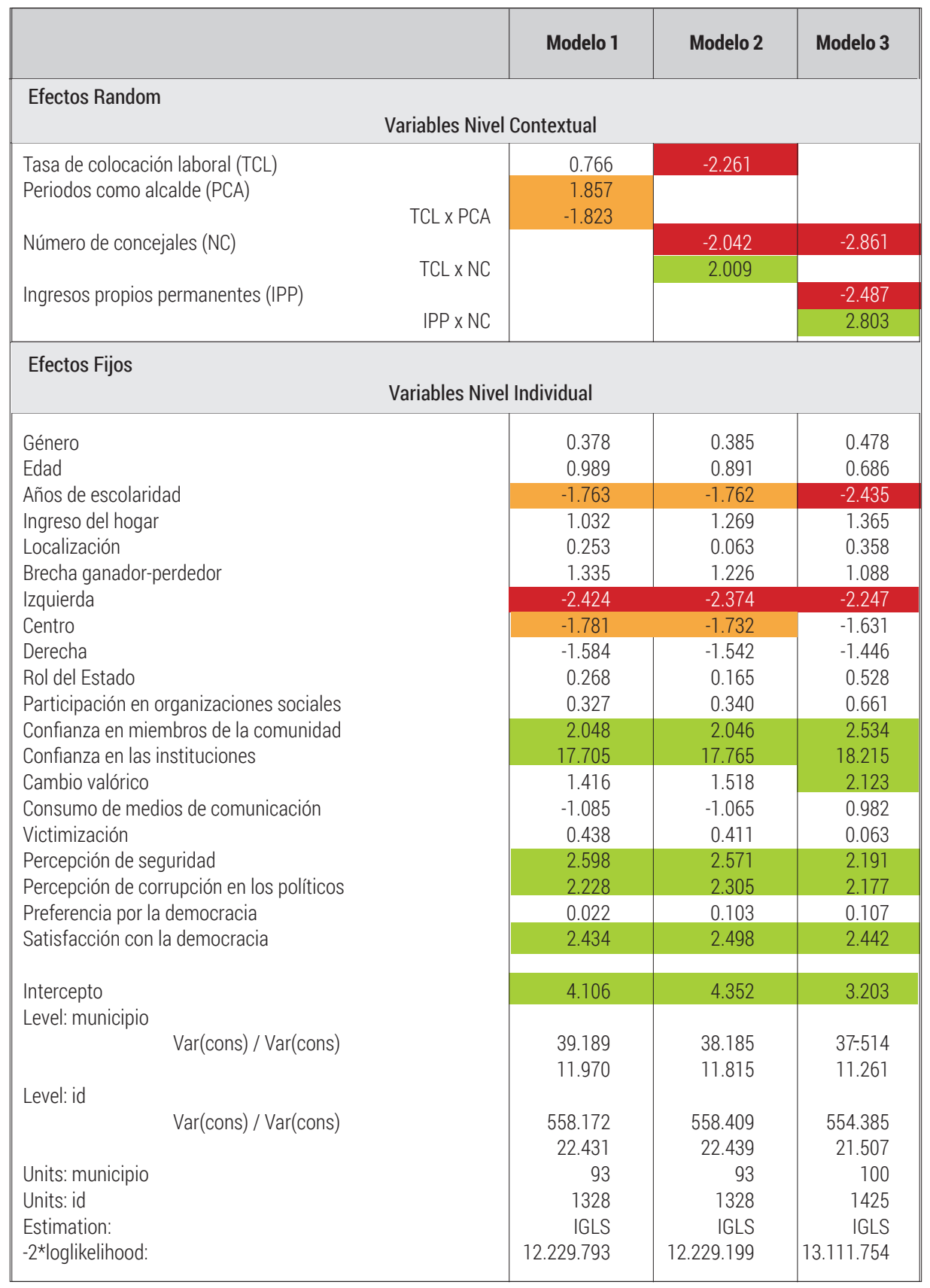

FUENTE: ELABORACIÓN PROPIA CON DATOS DE LAPOP 2016; SERVEL 2018; SINIM 2018. 


\section{O N C L U S I O N E S}

El presente estudio ha analizado la confianza en las municipalidades en Chile. Para ello, se optó por una estrategia de investigación de análisis multinivel con la finalidad de testear si las capacidades institucionales de las municipalidades inciden en la confianza política que las personas depositan en ellas y, de existir tal influencia, cómo es dicha relación. El argumento principal es que las municipalidades que presenten un mayor nivel de capacidades institucionales (administrativas-técnicas, políticas y extractivas/fiscal) presentarán mayores niveles de confianza en el municipio por parte de los residentes de tales comunas.

Para medir los efectos mencionados, se formularon dos hipótesis. Respecto a la primera, los resultados generados en esta investigación suman evidencia a que no todas las capacidades institucionales aumentan el nivel de confianza en las municipalidades. De las variables políticas, el tamaño del Concejo Municipal resultó ser la variable contextual más relevante de los nueve modelos testeados, desplazando en importancia al número de periodos consecutivos de los alcaldes en ejercicio. Esto abre ciertas incógnitas, pues en la escasa literatura politológica chilena que trata sobre temáticas locales, los concejales suelen ser ignorados como parte del sistema político, siendo los alcaldes quienes capturan, en mayor medida, la atención de los investigadores. Investigaciones sobre nivel de incumbencia de los concejales, gobierno municipal dividido o relación de los votantes/ciudadanos con los concejales, son temas que surgen como complemento y profundización del tema de este artículo.

En referencia a las capacidades administrativas-técnicas y las extractivas/fiscal, el porcentaje de colocación laboral como los ingresos permanentes surgieron como factores explicativos relevantes a nivel contextual.

Sobrela segundahipótesis (efectos deinteracción), seentrega evidencia en favorque la combinación de capacidades presenta efectos moderadores a nivel municipal. De hecho, en el caso del tamaño del Concejo Municipal, éste ejerce efectos moderadores positivos, tanto con la colocación laboral como con los ingresos permanentes.

A nivel de limitaciones, es necesario seguir estudiando el comportamiento de factores contextuales como lo son las capacidades institucionales. Si las instituciones importan para el desarrollo de las personas, es coherente agregar los factores aquí testeados. Segundo, es posible efectuar estudios longitudinales desde una perspectiva multinivel, con el objeto de probar el comportamiento de las capacidades institucionales y las de apoyo político a través del tiempo. Tercero, es necesario avanzar hacia estudios de método mixto en el caso de estudios políticos, pues hay dinámicas que los números no ven ni pueden ver. 


\section{RE F E R E N C I A S}

Anderson, C. J., \& Guillory, C. A. (1997). Political institutions and satisfaction with democracy: A cross-national analysis of consensus and majoritarian systems. American Political Science Review, 91(01), 66-81.

Anderson, C., Blais, A., Bowler, S., Donovan, T., \& Listhaug, O. (2005). Losers' consent: Elections and democratic legitimacy. Oxford: Oxford University Press.

Booth, J. A., \& Seligson, M. A. (2009). The legitimacy puzzle in Latin America: Political support and democracy in eight nations. Cambridge University Press.

Bühlmann, M. (2012). Municipal identity. A multilevel analysis of the determinants of individual attachment to municipalities. Zeitschrift für Vergleichende Politikwissenschaft, 6, 149-175.

Centro UC Políticas Públicas (2016a). Confianza municipal. Percepciones desde la escala local. Pontificia Universidad Católica de Chile. Santiago, Chile.

Centro UC Políticas Públicas (2016b). Informe de Resultados: Encuesta Nacional de Percepciones Ciudadanas sobre Municipios. Pontificia Universidad Católica de Chile. Santiago, Chile.

Clarke, P. (2008). When can group level clustering be ignored? Multilevel models versus single-level models with sparse data. Journal of Epidemiology and Community Health, 62(8), 752-758.

Completa, E. R. (2017). Capacidad estatal ¿Qué tipo de capacidades y para qué tipo de Estado? POSTData: Revista de Reflexión y Análisis Político, 22(1), 111-140.

Criado, H., \& Herreros, F. (2007). Political support: Taking into account the institutional context. Comparative Political Studies, 40(12), 1511-1532.

Dalton, R. (1999). Political Support in Advanced Industrial Democracies. In Norris, P. Critical citizens: Global support for democratic government (pp. 57-77). Oxford: Oxford Univ. Press.

Dalton, R. (2004). Democratic Challenges, Democratic Choices: The Erosion of Political Support in Advanced Industrial Democracies (Comparative Politics). Oxford: Oxford University Press, UK.

Dalton, R. J. (2006). Citizen politics: Public opinion and political parties in advanced industrial democracies. Washington, D.C: CQ Press.

Easton, D. (1957). An Approach to the Analysis of Political Systems. World Politics, 9 (3), 383-400.

Easton, D. (1965). A systems analysis of political life. New York: Wiley. 
Easton, D. (1975). A Re-assessment of the Concept of Political Support. British Journal of Political Science, 5(4), 435-457.

Easton, D. (1976). Theoretical Approaches to Political Support. Canadian Journal of Political Science / Revue Canadienne De Science Politique, 9(3), 431-448.

Freigedo, M., Fuentes, G., \& Milanesi, A. (2015). Capacidades estatales y regulación en asociaciones público-privadas: desafíos para las nuevas estrategias de desarrollo. Revista del CLAD Reforma y Democracia, (63).

Fuchs, D., \& Klingemann, H. (1995). Citizens and the State: A Changing Relationship? In Klingemann, H.-D., \& Fuchs, D. Citizens and the state. (pp. 1-23) Oxford: Oxford University Press, 1-23.

Fuchs, D., Guidorossi, G., \& Svensson, P. (1995). Support for the Democratic System. In Klingemann, H.-D., \& Fuchs, D. (1995). Citizens and the state. Oxford: Oxford University Press., 323-353.

Fuentes-González. (2018). Democracy and incumbency: A mixed method strategy to understand political support from the results of deputies' elections in Chile (Unpublished PhD thesis). Manchester School of Social Science, Manchester, UK.

Gilio, A. (2016). Desarrollo de capacidades estatales para gobiernos locales: dimensiones e indicadores para su diagnóstico. Revista del CLAD Reforma y Democracia, (66).

Goldstein, \& Silver. (1989) Multilevel and multivariatem odels in survey analysis, in: C. J. Skinnerd, Holt and T. M. F. Smith (Eds) Analysis of Complex Surveys, Chichester, Wiley.

Goldstein, H. (1995). Multilevel statistical models. London: Arnold.

Grindle, Merilee S. (1996). Challenging the State: Crisis and Innovation in Latin America and Africa, Cambridge: Cambridge University Press.

Guarda, P. (2017). Personal a honorarios en municipios se duplicó entre 2006 y 2016. La Tercera. Disponible en: https://www.latercera.com/noticia/personal-honorarios-municipios-se-duplico-2006-2016 (Accesado el 27 de noviembre de 2018).

Hernández, J. (2016). Índice Institucional para el Gobierno Abierto Municipal. Instituto Chileno de Estudios Municipales (ICHEM). Facultad de Ciencias Sociales y Humanidades. Universidad Autónoma de Chile. Santiago, Chile.

Hox, J. J. (2002). Multilevel analysis: Techniques and applications. Mahwah, N.J: Lawrence Erlbaum Publishers.

Klingemann, H. (1999). Mapping Political Support in the 1990s: A Global Analysis. In Norris, P. Critical citizens: Global Support for Democratic Government (pp. 31-56). Oxford: Oxford University Press.

Listhaug, O. (1995). The Dynamics of Trust in Politicians. In Citizens and the State (pp. 261-297). Oxford: Oxford University Press. 
Luna, J. P. (2014). Segmented representation: Political party strategies in unequal democracies. Oxford: Oxford University Press.

Luna, J. P., Toro, S., Salas, V., \& Jaramillo-Brun, N. (2012). Cultura politica de la democracia en Chile y en las Américas, 2012: Hacia la igualdad de oportunidades. Santiago: LAPOP/ Pontificia Universidad Católica.

Luna, J. P., Zechmeister, E. J., \& Seligson, M. A. (2010). Cultura política de la democracia en Chile, 2010: Consolidación democrática en las Américas en tiempos difíciles. Santiago, Chile: Vanderbilt University.

MacKuen, M., \& Brown, C. (1987). Political context and attitude change. American Political Science Review, 81, 2.

Mishler, W., \& Rose, R. (1997). Trust, distrust and skepticism: Popular evaluations of civil and political institutions in post-communist societies. The Journal of Politics, 59(2), 418-451.

Morales, Q. M. (2008). Evaluando la confianza institucional en Chile: Una mirada desde los resultados LAPOP. Revista De Ciencia Política, 28, 3, 161-186.

Netelenbos, B. (2016). Political Legitimacy beyond Weber: An Analytical Framework. London: Palgrave Macmillan.

Newton, K., \& Norris, P. (2000). Confidence in public institutions. In Pharr, S. J., \& Putnam, R. D. Disaffected democracies: What's troubling the trilateral countries? (pp. 5273) Princeton, NJ: Princeton University Press.

Norris, P. (1999b). Institutional Explanations for Political Support. In Norris, P. Critical Citizens: Global Support for Democratic Governance (pp. 217-235). Oxford University Press.

Norris, P. (2011). Democratic deficit: Critical citizens revisited. Cambridge: Cambridge University Press.

Norris, P. (2017). The conceptual framework of political support. In Zmerli, S., \& van der Meer, T. W. (Eds.). Handbook on Political Trust (pp. 19-32). Edward Elgar Publishing.

Norris. P. (1999a). Introduction: the growth of critical citizens. In Norris, P. Critical citizens: Global Support for Democratic Government (pp. 1-27). Oxford University Press.

Paterson, L., \& Goldstein, H. (1991). New statistical methods for analysing social structures: an introduction to multilevel models. British Educational Research Journal, 17(4), 387-393.

Putnam, R. D. (2000). Bowling alone: The collapse and revival of American community. New York: Simon \& Schuster.

Putnam, R. D., Leonardi, R., \& Nanetti, R. Y. (1993). Making democracy work: Civic traditions in modern Italy. Princeton: Princeton University Press. 
Putnam, R. D., Pharr, S. J., \& Dalton, R. J. (2000). Introduction: What's Troubling the Trilateral Democracies? In Pharr, S. J., \& Putnam, R. D. (2000). Disaffected democracies: What's troubling the trilateral countries? (pp. 3 - 27). Princeton, NJ: Princeton University Press.

Rahn, W. M., \& Rudolph, T. J. (2005). A Tale of Political Trust in American Cities. Public Opinion Quarterly, 69, 4, 530-560.

Rasbash, J. (2008). Module 4: Multilevel structures and classifications. Centre for Multilevel Modelling. University of Bristol.

Rasbash, J., Steele, F., Browne, W. J., Goldstein, H., \& Charlton, C. (2015). A user's guide to MLwiN. Centre for Multilevel Modelling, University of Bristol, UK.

Repetto, F. (2004). Capacidad Estatal: requisito para el mejoramiento de la Política Social en América Latina. Departamento de Integración y Programas Regionales Instituto Interamericano para el Desarrollo Social. Banco Interamericano de Desarrollo. Serie de Documentos de Trabajo I-52.

Rodríguez-Gustá, A. L. (2004). Capacidades estatales: reflexiones en torno a un programa de investigación. IX Congreso Internacional del CLAD sobre la Reforma del Estado y de la Administración Pública, Madrid, España.

Salinas, E., \& Booth, J. A. (2011). Micro-social and contextual sources of democratic attitudes in Latin America. Journal of Politics in Latin America, 3(1), 29-64.

Segovia, C., Haye, A., Gonzalez, R., Manzi, J., \& Carvacho, H. (December 01, 2008). Confianza en instituciones políticas en Chile: Un modelo de los componentes centrales de juicios de confianza. Revista de Ciencia Política, 28, 2, 39-60.

SINIM. (2018). Guía de Búsqueda de Información. Departamento de Finanzas Municipales, División de Municipalidades, Subsecretaría de Desarrollo y Administración Regional. Santiago, Chile.

Snijders, T., and Bosker, R. (1999). Multilevel modeling: An introduction to basic and advanced multilevel modeling. London: Sage Publications.

Thomassen, J. (1995). Support for democratic values. In Klingemann, H.-D., \& Fuchs, D. Citizens and the state (pp. 383-416). Oxford: Oxford University Press. 